\title{
From convective to stellar dynamos
}

\author{
Axel Brandenburg ${ }^{1,2}$, Petri J. Käpylä ${ }^{1,3}$, Maarit J. Korpi ${ }^{3}$ \\ ${ }^{1}$ NORDITA, Roslagstullsbacken 23, SE-10691 Stockholm, Sweden \\ ${ }^{2}$ Department of Astronomy, Stockholm University, SE-10691 Stockholm, Sweden \\ ${ }^{3}$ Department of Physics, PO Box 64, FI-00014 University of Helsinki, Finland
}

\begin{abstract}
Convectively driven dynamos with rotation generating magnetic fields on scales large compared with the scale of the turbulent eddies are being reviewed. It is argued that such fields can be understood as the result of an $\alpha$ effect. Simulations in Cartesian domains show that such large-scale magnetic fields saturate on a time scale compatible with the resistive one, suggesting that the magnitude of the $\alpha$ effect is here still constrained by approximate magnetic helicity conservation. It is argued that, in the absence of shear and/or any other known large-scale dynamo effects, these simulations prove the existence of turbulent $\alpha^{2}$-type dynamos. Finally, recent results are discussed in the context of solar and stellar dynamos.
\end{abstract}

Keywords. MHD - turbulence - Sun: magnetic fields

\section{Introduction}

Stars with outer convection zones are known to display magnetic activity, often in a cyclic fashion like in the Sun. Such activity can generally be explained by a turbulent dynamo influenced by rotation and stratification to produce the anisotropies required for the generation of large-scale magnetic fields. The basic theory is reasonably well understood (Moffatt 1978; Parker 1979; Krause \& Rädler 1980), but there continues to be substantial controversy until the present day. A major stumbling block has been the understanding of what is known as catastrophic quenching (Vainshtein \& Cattaneo 1992; Cattaneo \& Hughes 1996) and resistively limited saturation (Brandenburg 2001), as well as the very existence of the $\alpha$ effect in convection even without nonlinearity (Cattaneo \& Hughes 2006; Hughes \& Cattaneo 2008).

The first two issues have been reviewed in detail by Brandenburg \& Subramanian (2005). The purpose of the present paper is to review recent progress concerning convective dynamos. However, in view of applications to solar and stellar dynamos, it is important to realize that we are still lacking simulations that reproduce the salient features of the solar dynamo. We should therefore keep our eyes open for new phenomena that may emerge as simulations become more realistic.

\section{Excitation conditions of small-scale and large-scale dynamos}

Small-scale and large-scale dynamos are quite different in nature. The difference becomes most evident in the nonlinearly saturated regime, provided one allows for what we call scale separation, which means that the size of the domain is large compared with the scale of the largest (energy-carrying) eddies of the turbulence. In Figure 1 we show spectra highlighting the remarkable difference between the two types of dynamos. Conversely, if there is insufficient scale separation, a large-scale dynamo becomes impossible and both types of simulations would look very similar, as has been demonstrated by Haugen et al. (2004); see their Fig. 23. 

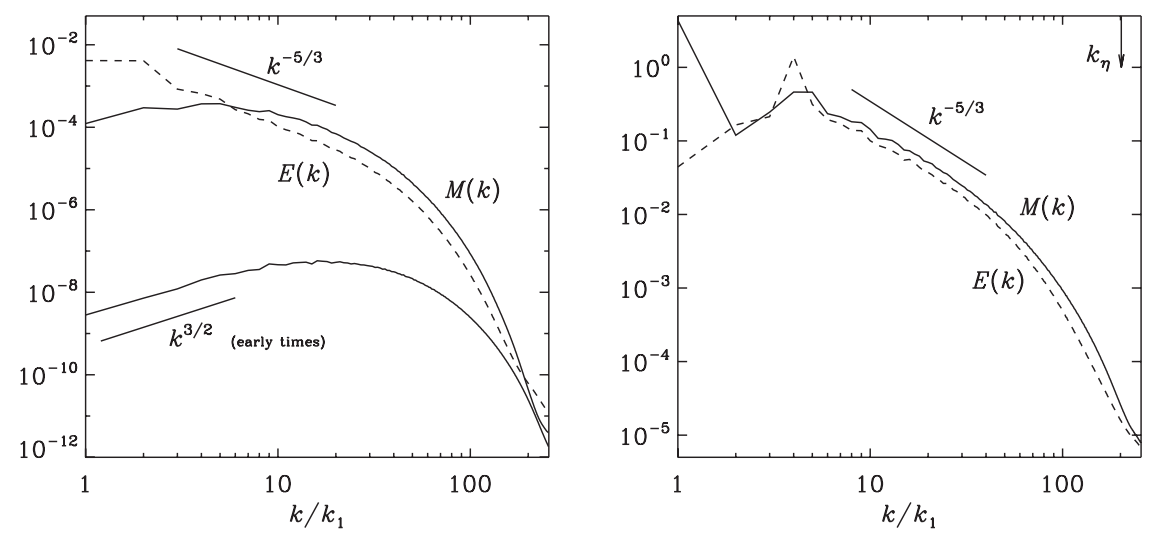

Figure 1. Kinetic and magnetic energy spectra in a turbulence simulation without net helicity (left) and with net helicity (right) for a magnetic Prandtl number of unity and a mesh size is $512^{3}$ meshpoints. Notice the pronounced peak of $M(k)$ at $k=k_{1}$ in the case with helicity. Adapted from Brandenburg \& Subramanian (2005) and Brandenburg (2009), respectively.

There can be different types of large-scale dynamos. The most frequently studied ones are the $\alpha^{2}$ and $\alpha \Omega$ type dynamos. These are dynamos that can produce large-scale magnetic fields owing to the presence of kinetic helicity in the turbulence, giving rise to an $\alpha$ effect. The presence of shear can further modify the dynamo, making it usually easier to excite and favoring oscillatory over non-oscillatory solutions.

Shear can be a typical result of rotation of a gaseous body in the presence of anisotropic turbulence (Rüdiger 1980, 1989). Shear alone is often found to give rise to large-scale fields - even if the turbulence is non-helical (Brandenburg 2005; Yousef et al. 2008a,b; Brandenburg et al. 2008). The nature of such dynamo action is still a matter of debate and ranges from incoherent $\alpha \Omega$ dynamos (Vishniac \& Brandenburg 1997; Proctor 2007) to shear-current dynamos (Rogachevskii \& Kleeorin 2003, 2004).

Returning to the $\alpha^{2}$ and $\alpha \Omega$ dynamos, it is important to realize that their excitation conditions are generally quite different. The onset of small-scale dynamo action depends generally on the value of the magnetic Reynolds number,

$$
\operatorname{Re}_{M}=u_{\mathrm{rms}} / \eta k_{\mathrm{f}},
$$

where $u_{\mathrm{rms}}$ is the typical rms velocity of the turbulence, $\eta$ is the microscopic magnetic diffusivity, and $k_{\mathrm{f}}$ is the forcing or integral wavenumber, i.e. the wavenumber of the energy-carrying motions. This is roughly where the peak of the energy spectrum is located. The critical value, $R_{\mathrm{m}, \mathrm{crit}}$, above which dynamo action commences, depends on the value of the magnetic Prandtl number, $\operatorname{Pr}_{M}=\nu / \eta$, where $\nu$ is the microscopic kinematic viscosity and is about 35 for $\operatorname{Pr}_{M}=1$ (Novikov et al. 1983; Subramanian 1999; Haugen et al. 2004), but increases to values around and above 400 for $\operatorname{Pr}_{M}$ somewhere between 0.2 and 0.1 (Schekochihin et al. 2005). There is now also evidence that $R_{\mathrm{m}, \mathrm{crit}}$ may actually show a peak at $\operatorname{Pr}_{M}=0.1$ and might then drop to slightly lower values for $\operatorname{Pr}_{M}=0.05$ and below (Iskakov 2007). This unusual behavior is connected with a change of the "roughness" of the velocity field (Boldyrev \& Cattaneo 2004) and the occurrence of a bottleneck effect in the velocity spectrum (Falkovich 1994; Dobler et al. 2003), which means that the velocity has maximum roughness for $\operatorname{Pr}_{M} \approx 0.1$ when the resistive scale coincides with the position of the bottleneck.

The situation is quite different with large-scale dynamos that operate completely independently of the value of $\operatorname{Pr}_{M}$, as long as $\operatorname{Re}_{M}$ is large enough. Already in Brandenburg 



Figure 2. Snapshots of $B_{y}$ in the early phase (left) and saturated phase (right) of a convective dynamo with shear. Adapted from Käpylä et al. (2008); see also http://www.helsinki.fi/ kapyla/movies.html

(2001) the critical value of $\mathrm{Re}_{M}$ was found to be around unity regardless of whether $\operatorname{Pr}_{M}=1$ or 0.1 . This finding was then extended by Mininni (2007) and Brandenburg (2009), who demonstrated dynamo action down to $\operatorname{Pr}_{M}=0.005$ and 0.001 , respectively, or up to $\operatorname{Pr}_{M}=1000$ (Brandenburg 2011). The conclusion is that large-scale dynamo action depends solely on the dynamo number, which is given by $D=C_{\alpha}$ for $\alpha^{2}$ dynamos and by $D=C_{\alpha} C_{S}$ for $\alpha \Omega$ (or $\alpha$-shear) dynamos. Here,

$$
C_{\alpha}=\alpha_{0} / \eta_{\mathrm{T}} k_{1} \quad \text { and } \quad C_{S}=S / \eta_{\mathrm{T}} k_{1}^{2},
$$

where $\eta_{\mathrm{T}}=\eta_{\mathrm{t}}+\eta$ is the sum of turbulent and microscopic magnetic diffusivities, $\alpha_{0}$ is a typical value of the $\alpha$ effect, and $S$ is the shear rate (i.e. a typical value of the velocity gradient). For $C_{\alpha}$ and $C_{S}$, we use standard estimates:

$$
\alpha_{0} \approx-\frac{\tau}{3} \overline{\boldsymbol{\omega} \cdot \boldsymbol{u}} \approx-\frac{\epsilon_{\mathrm{f}}}{3 u_{\mathrm{rms}} k_{\mathrm{f}}} k_{\mathrm{f}} u_{\mathrm{rms}}^{2}=-\frac{1}{3} \epsilon_{\mathrm{f}} u_{\mathrm{rms}}
$$

where $\epsilon_{\mathrm{f}}=\overline{\boldsymbol{\omega} \cdot \boldsymbol{u}} / k_{\mathrm{f}} u_{\mathrm{rms}}^{2}$ is a measure of the relative kinetic helicity, $\tau=\left(u_{\mathrm{rms}} k_{\mathrm{f}}\right)^{-1}$ is the turnover time, and

$$
\eta_{\mathrm{t}} \approx \frac{\tau}{3} \overline{\boldsymbol{u}^{2}} \approx u_{\mathrm{rms}} / 3 k_{\mathrm{f}}
$$

With this we find

$$
C_{\alpha}=-\frac{\frac{1}{3} \epsilon_{\mathrm{f}} u_{\mathrm{rms}} / k_{1}}{u_{\mathrm{rms}} / 3 k_{\mathrm{f}}+\eta}=-\iota \epsilon_{\mathrm{f}} \frac{k_{\mathrm{f}}}{k_{1}}
$$

where

$$
\iota=1 /\left(1+3 \operatorname{Re}_{M}^{-1}\right)
$$

is a correction factor that is close to unity for $\operatorname{Re}_{M} \gg 1$. Furthermore, we have

$$
C_{S}=\frac{S / k_{1}^{2}}{u_{\mathrm{rms}} / 3 k_{\mathrm{f}}+\eta}=\frac{3 \iota S}{u_{\mathrm{rms}} k_{\mathrm{f}}}\left(\frac{k_{\mathrm{f}}}{k_{1}}\right)^{2}=3 \iota \mathrm{Sh}\left(\frac{k_{\mathrm{f}}}{k_{1}}\right)^{2},
$$

where we have defined the shear parameter $\mathrm{Sh}=S / u_{\mathrm{rms}} k_{\mathrm{f}}$. Note that, especially in the presence of shear, the possibility of dynamo action is strongly connected with the scale separation ratio. Indeed,

$$
D=-3 \iota \epsilon_{\mathrm{f}} \operatorname{Sh}\left(\frac{k_{\mathrm{f}}}{k_{1}}\right)^{3}
$$

depends cubicly on the scale separation ratio. This explains why $\alpha \Omega$ dynamos are often much easier to obtain than $\alpha^{2}$ dynamos. 


\section{Large-scale dynamos in Cartesian domains}

\subsection{Dynamos of $\alpha \Omega$ type}

Given the alarming signs of earlier investigations by Cattaneo \& Hughes (2006) and Hughes \& Cattaneo (2008), it was quite unclear whether the $\alpha$ effect even exists in simulations with convection. At the time, several possible reasons were put forward, including the absence of stratification; see, for example, Brandenburg (2009). In the wake of this initial frustration, it was quite surprising when large-scale dynamo action was found in rotating convection in the presence of shear (Käpylä et al. 2008); see Figure 2. Similar results were obtained by Hughes \& Proctor (2009). This controversy was still ongoing at the conference "Turbulence and Dynamos" in Stockholm in March 2008† where Hughesł argued that no convective large-scale dynamos exist, while Käpylä $₫$ showed results from low Reynolds number convection with shear where large-scale fields were indeed obtained. In an attempt to clarify the still conflicting results regarding the actual value of $\alpha$ in rotating convection, Käpylä et al. (2010a) pointed out that for a nonuniform mean field, the mean current density cannot be neglected. In this case, the turbulent magnetic diffusivity contributes and explains the small net electromotive force measured by Hughes \& Proctor (2009) by imposing a uniform field.

\subsection{Dynamos of $\alpha^{2}$ type}

The simulations mentioned above do not provide conclusive evidence for the existence of an $\alpha$ effect in rotating convection, because it is in principle possible that the dynamo could be the result of an incoherent $\alpha \Omega$ dynamo or a shear-current dynamo. In the absence of shear, however, there is no viable alternative to an $\alpha^{2}$ dynamo. It is therefore important to consider the conceptually simpler case without imposed shear, as was also emphasized by Hughes et al. (2011), who noted that this was not done by Käpylä et al. (2010a), who just considered the case of a sinusoidal shear profile. For this reason, we discuss in the following the papers of Käpylä et al. (2009a) and, in particular, Käpylä et al. (2009b), where large-scale dynamo action was studied in non-shearing convection at sufficiently large Coriolis numbers.

Before trying to simulate an $\alpha^{2}$ dynamo for rotating convection, it is instructive to obtain guidance from numerically obtained measurements of the $\alpha$ and turbulent diffusivity tensors. This can be done using the test-field method (Schrinner et al. 2005, 2007), which has been applied to turbulence in a number of recent papers (Brandenburg 2005; Brandenburg et al. 2008). The result is shown in Figure 5. Using this method, Käpylä et al. (2009a) noted that the magnitude of the relevant components of the $\alpha$ tensor vary only weakly with Coriolis number, $\mathrm{Co}=2 \Omega / u_{\mathrm{rms}} k_{\mathrm{f}}$, where $\Omega$ is angular velocity, while $\eta_{\mathrm{t}}$ diminishes with increasing values of Co. This was a clear indication that dynamos of $\alpha^{2}$ type might become possible once Co is large enough. We emphasize this point, because it is one of the several examples where mean-field theory has proven its predictive power.

Consequently, in a subsequent investigation, Käpylä et al. (2009b) carried out simulations for large enough values of Co and did indeed find dynamo action of large-scale type when Co $\gtrsim 10$. The large-scale field became even more pronounced as the aspect ratio was increased. In Figure 3 we present horizontal spectra of magnetic and kinetic energies. What is important here is the fact that, even though the magnetic energy is less (by factor 5 ) than the kinetic energy at what we estimate to be $k_{\mathrm{f}}\left(\right.$ about $5 k_{1}$ ), the magnetic energy strongly exceeds the kinetic energy at the scale of the domain. This seems to

$\dagger$ http://agenda.albanova.se/conferenceDisplay.py?conf Id=325

$\ddagger$ http://videos.nordita.org/conference/Turbulence2008/hires/March17/Part1. WMV

đ http://videos.nordita.org/conference/Turbulence2008/hires/March19/Part5. WMV 

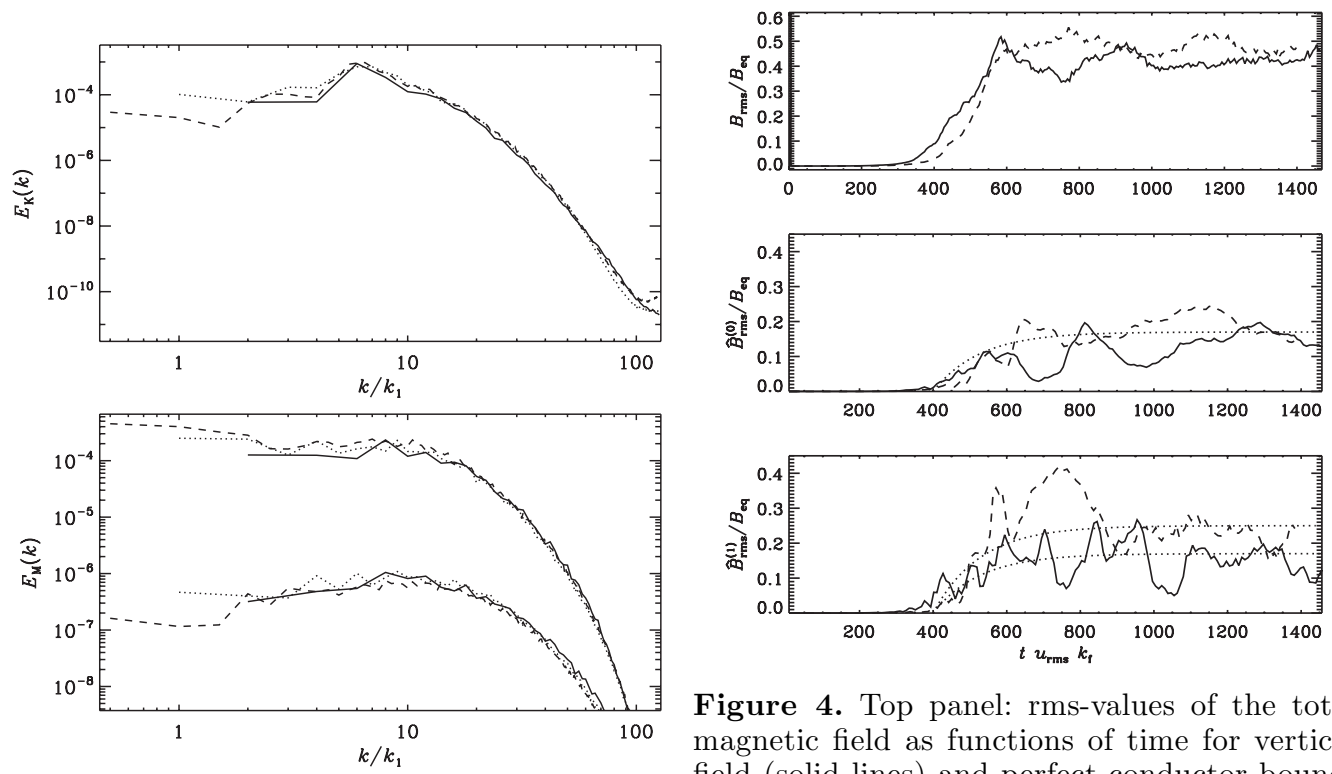

Figure 4. Top panel: rms-values of the total magnetic field as functions of time for vertical field (solid lines) and perfect conductor bound-

Figure 3. Two-dimensional power spectra of velocity (upper panel) and magnetic field (lower panel) as functions of system size. In the lower panel the upper curves show the spectra from the saturated state whereas the lower curves show the spectra from the kinematic state multiplied by $10^{7}$. Adapted from Käpylä et al. (2009b).

ary conditions (dashed lines). The two lower panels show the sums of the rms-values of the Fourier amplitudes of $B_{x}$ and $B_{y}$ for $k / k_{1}=0$ (middle panel) and $k / k_{1}=1$ (bottom panel). The dotted lines in the two lower panels show a saturation predictor according to the model of Brandenburg (2001). Adapted from Käpylä et al. (2009b).

exclude alternative explanations whereby the magnetic field at smaller wavenumbers might just be a trivial result of diffusion in wavenumber space. Instead, we argue that this is strong evidence for the physical reality of an $\alpha^{2}$ dynamo driven by rotating convection.

In agreement with virtually all earlier work on large-scale dynamos of $\alpha^{2}$ type the saturation time of the dynamo is comparable with the resistive time. Indeed, Brandenburg (2001) found that in the absence of strong magnetic helicity fluxes, the saturation of an $\alpha^{2}$ dynamo follows a switch-on behavior where, after saturation, the mean field is given by

$$
\frac{\overline{\boldsymbol{B}}^{2}}{B_{\mathrm{eq}}^{2}} \approx \frac{k_{\mathrm{f}}}{k_{\mathrm{m}}}\left[1-e^{-2 \eta k_{\mathrm{m}}^{2}\left(t-t_{\mathrm{s}}\right)}\right] .
$$

This is also seen in the present case; see Figure 4, where we overplot the prediction from Equation (3.1).

It is likely that diffusive magnetic helicity fluxes are present in the convection simulations discussed above (Brandenburg et al. 2009). Those fluxes could in principle give rise to faster saturation times than what is seen in Figure 3.1. This question has been addressed quantitatively by Mitra et al. (2010a) and Hubbard \& Brandenburg (2010), who note that at the magnetic Reynolds numbers accessible so far, diffusive helicity fluxes are still quite weak compared with the resistive processes. Based on their scalings for different values of $\operatorname{Re}_{M}$, they estimate that resistive saturation effects would only begin to be alleviated for $\mathrm{Re}_{M}$ well in excess of values around 1000 or even $10^{4}$; see also Fig. 10 of Candelaresi et al. (2011). Convection simulations with closed magnetic boundaries do 



Figure 5. Normalized profiles of $\alpha$ (left panel) and $\eta_{\mathrm{t}}$ (right panel) from kinematic test field simulations. The vertical dotted lines at $z / d=0$ and $z / d=1$ indicate the base and top of the convectively unstable layer, respectively. Adapted from Käpylä et al. (2009b).

seem to suffer from catastrophic quenching at least up to $\operatorname{Re}_{M} \approx 240$ (Käpylä et al. 2010b). Reaching much higher values to study this issue further in the near future is not possible. While this is certainly somewhat discouraging news, it does highlight the importance of studying detailed scaling properties of large-scale dynamos rather than producing solitary examples of dynamos at large resolution, hoping that they represent the Sun.

\section{Bi-helical magnetic field}

An important property of $\alpha$ effect dynamos is the fact that they produce bi-helical magnetic fields. This means that one expects to see magnetic helicity fluxes with opposite signs at small and large scales. While this is now well established theoretically (Blackman \& Brandenburg 2003; Yousef \& Brandenburg 2003), there is still no widespread observational evidence for this. Large-scale magnetic helicity can be estimated using synoptic maps of the azimuthally averaged radial magnetic field of the Sun; see Fig. 1 of Brandenburg et al. (2003). Small-scale magnetic helicity fluxes have been inferred from magnetic field measurements in active regions and coronal mass ejections (Berger \& Ruzmaikin 2000).

There is also some evidence from measurements of magnetic helicity in the solar wind. Using the assumption of homogeneity, Matthaeus et al. (1982) were able to determine magnetic helicity spectra for the solar wind. Preliminary analysis of more recent solar wind data from the Ulysses spacecraft does indeed suggest that the field in the solar wind is also bi-helical. Further details on this are presented in a dedicated publication (Brandenburg et al. 2011).

\section{Concluding remarks}

We still do not know exactly how the solar dynamo works. If it is of $\alpha \Omega$ type, given that the $\alpha$ effect is positive in the northern hemisphere, and using the fact that radial shear is positive in the bulk of the convection zone, one would expect poleward migration of the dynamo wave. This is also what three-dimensional simulations in spherical shells have shown repeatedly over several decades starting with the early work of Gilman (1983), and now again in the spherical wedge simulations of Käpylä et al. (2010c). For rapid rotation, 
however, polarity flips of toroidal magnetic field can also occur more abruptly, as has been demonstrated by Brown et al. (2010) and Ghizaru et al. (2010), which is beginning to be reminiscent of polarity reversals in the geodynamo (Glatzmaier \& Roberts 1995), but is different from what we know about the solar dynamo. In this connection it is worth recalling yet another recent surprise: oscillatory solutions with equatorward migration are also possible in the absence of any differential rotation provided the dynamo is somehow bounded between highly conducting media at high latitudes (Mitra et al. 2010b). It is obviously unclear whether this has any bearing on the solar dynamo, but it reminds us of the possibility of surprises.

Other proposals for how the solar dynamo might work include the flux transport dynamo (Durney 1995; Choudhuri et al. 1995; Dikpati \& Charbonneau 1999), and the possibility of a dynamo shaped by the negative radial angular velocity gradient in the near-surface shear layer (Brandenburg 2005). Neither of these two scenarios have been seen in three-dimensional turbulence simulations. The former suffers from the difficulty of obtaining a sufficiently coherent meridional circulation that does not break up into smaller circulation patterns, while the latter may suffer from the difficulty of resolving the small-scale turbulence in the near-surface shear layer. A possible step forward might therefore be a combined effort utilizing a range of different simulations in spherical and Cartesian geometries on the one hand, and improved mean-field theory on the other. Clearly, in order to improve mean-field theory it is essential to seek guidance from direct simulations, as has already been done in recent years with increasing success.

\section{Acknowledgements}

We acknowledge the allocation of computing resources provided by the Swedish National Allocations Committee at the Center for Parallel Computers at the Royal Institute of Technology in Stockholm and the National Supercomputer Centers in Linköping as well as the Norwegian National Allocations Committee at the Bergen Center for Computational Science and the computing facilities hosted by CSC - IT Center for Science Ltd. in Espoo, Finland, who are administered by the Finnish Ministry of Education. This work was supported in part by the European Research Council under the AstroDyn Research Project 227952, the Swedish Research Council grant 621-2007-4064, and the Finnish Academy grants 121431, 136189, and 112020.

\section{References}

Berger, M. A. \& Ruzmaikin, A. 2000, J. Geophys. Res., 105, 10481

Blackman, E. G. \& Brandenburg, A. 2003, ApJ, 584, L99

Boldyrev, S. \& Cattaneo, F. 2004, Phys. Rev. Lett., 92, 144501

Brandenburg, A. 2001, ApJ, 550, 824

Brandenburg, A. 2005, ApJ, 625, 539

Brandenburg, A. 2005, Astron. Nachr., 326, 787

Brandenburg, A. 2009, ApJ, 697, 1206

Brandenburg, A. 2009, Space Sci. Rev., 144, 87

Brandenburg, A. 2011, Astron. Nachr., 332, 51

Brandenburg, A. \& Subramanian, K. 2005, Phys. Rep., 417, 1

Brandenburg, A., Blackman, E. G., \& Sarson, G. R. 2003, Adv. Space Sci., 32, 1835

Brandenburg, A., Rädler, K.-H., Rheinhardt, M., \& Käpylä, P. J. 2008, ApJ, 676, 740

Brandenburg, A., Candelaresi, S., \& Chatterjee, P. 2009, MNRAS, 398, 1414

Brandenburg, A., Subramanian, K., Balogh, A., \& Goldstein, M. L. 2011, arXiv:1101.1709

Brown, B. P., Browning, M. K., Brun, A. S., Miesch, M. S., \& Toomre, J. 2010, ApJ, 711, 424

Candelaresi, S., Hubbard, A., Brandenburg, A., \& Mitra, D. 2011, Phys. Plasmas, 18, 012903 
Cattaneo, F. \& Hughes, D. W. 1996, Phys. Rev. E, 54, R4532

Cattaneo, F. \& Hughes, D. W. 2006, J. Fluid Mech., 553, 401

Choudhuri, A. R., Schüssler, M. \& Dikpati, M. 1995, A\&A, 303, L29

Dikpati, M. \& Charbonneau, P. 1999, ApJ, 518, 508

Dobler, W., Haugen, N. E. L., Yousef, T. A. \& Brandenburg, A. 2003, Phys. Rev. E, 68, 026304

Durney, B. R. 1995, Solar Phys., 160, 213

Falkovich, G. 1994, Phys. Fluids, 6, 1411

Ghizaru, M., Charbonneau, P. \& Smolarkiewicz, P. K. 2010, ApJ, 715, L133

Gilman, P. A. 1983, ApJS, 53, 243

Glatzmaier, G. A. \& Roberts, P. H. 1995, Nature, 377, 203

Haugen, N. E. L., Brandenburg, A. \& Dobler, W. 2004, Phys. Rev. E, 70, 016308

Hubbard, A. \& Brandenburg, A. 2010, Geophys. Astrophys. Fluid Dyn., 104, 577

Hughes, D. W. \& Cattaneo, F. 2008, J. Fluid Mech., 594, 445

Hughes, D. W. \& Proctor, M. R. E. 2009, Phys. Rev. Lett., 102, 044501

Hughes, D. W., Proctor, M. R. E. \& Cattaneo, F. 2011, arXiv:1103.0754

Iskakov, A. B., Schekochihin, A. A., Cowley, S. C., McWilliams, J. C. \& Proctor, M. R. E. 2007, Phys. Rev. Lett., 98, 208501

Käpylä, P. J., Korpi, M. J., \& Brandenburg, A. 2008, A\&A, 491, 353

Käpylä, P. J., Korpi, M. J., \& Brandenburg, A. 2009a, A\&A, 500, 633

Käpylä, P. J., Korpi, M. J., \& Brandenburg, A. 2009, ApJ, 697, 1153

Käpylä, P. J., Korpi, M. J., \& Brandenburg, A. 2010a, MNRAS, 402, 1458

Käpylä, P. J., Korpi, M. J., \& Brandenburg, A. 2010b, A\&A, 518, A22

Käpylä, P. J., Korpi, M. J., \& Brandenburg, A., Mitra, D., \& Tavakol, R. 2010b, Astron. Nachr., 331,73

Krause, F. \& Rädler, K.-H. 1980, Mean-field magnetohydrodynamics and dynamo theory (Pergamon Press, Oxford)

Matthaeus, W. H., Goldstein, M. L., \& Smith, C. 1982, Phys. Rev. Lett., 48, 1256

Mininni, P. D. 2007, Phys. Rev. E, 76, 026316

Mitra, D., Candelaresi, S., Chatterjee, P., Tavakol, R., \& Brandenburg, A. 2010a, Astron. Nachr., 331,130

Mitra, D., Tavakol, R., Käpylä, P. J., \& Brandenburg, A. 2010b, ApJ, 719, L1

Moffatt, H.K. 1978, Magnetic field generation in electrically conducting fluids (Cambridge University Press, Cambridge)

Novikov, V. G., Ruzmaikin, A. A., \& Sokoloff, D. D. 1983, Sov. Phys. JETP, 58, 527

Parker, E. N. 1979, Cosmical magnetic fields (Oxford University Press, New York)

Proctor, M. R. E. 2007, MNRAS, 382, L39

Rogachevskii, I. \& Kleeorin, N. 2003, Phys. Rev. E, 68, 036301

Rogachevskii, I. \& Kleeorin, N. 2004, Phys. Rev. E, 70, 046310

Rüdiger, G. 1980, Geophys. Astrophys. Fluid Dyn., 16, 239

Rüdiger, G. 1989, Differential rotation and stellar convection: Sun and solar-type stars (Gordon \& Breach, New York)

Schekochihin, A. A., Haugen, N. E. L., Brandenburg, A., Cowley, S. C., Maron, J. L., \& McWilliams, J. C. 2005, ApJ, 625, L115

Schrinner, M., Rädler, K.-H., Schmitt, D., Rheinhardt, M. \& Christensen, U. 2005, Astron. Nachr., 326, 245

Schrinner, M., Rädler, K.-H., Schmitt, D., Rheinhardt, M., \& Christensen, U. R. 2007, Geophys. Astrophys. Fluid Dyn., 101, 81

Subramanian, K. 1999, Phys. Rev. Lett., 83, 2957

Subramanian, K. \& Brandenburg, A. 2006, ApJ, 648, L71

Vainshtein, S. I. \& Cattaneo, F. 1992, ApJ, 393, 165

Vishniac, E. T. \& Brandenburg, A. 1997, ApJ, 475, 263

Warnecke, J. \& Brandenburg, A. 2010, A\&A, 523, A19

Yousef, T. A. \& Brandenburg, A. 2003, A\&A, 407, 7

Yousef, T. A., Heinemann, T., Schekochihin, A. A., Kleeorin, N., Rogachevskii, I., Iskakov, A. B., Cowley, S. C. \& McWilliams, J. C. 2008a, Phys. Rev. Lett., 100, 184501 
Yousef, T. A., Heinemann, T., Rincon, F., Schekochihin, A. A., Kleeorin, N., Rogachevskii, I., Cowley, S. C., \& McWilliams, J. C. 2008b, Astron. Nachr., 329, 737

\section{Discussion}

M. Proctor: What do you do about the gauge when calculating magnetic helicity, and does it make any difference to the answers obtained?

A. Brandenburg: Yes, the magnetic helicity density is in general gauge-dependent. However, if there is sufficient scale separation between mean and fluctuating fields, the magnetic helicity density computed from the fluctuating field can be shown to be gaugeinvariant (Subramanian \& Brandenburg 2006). Hubbard \& Brandenburg (2010) have recently confirmed this in a simulation where the magnetic helicity from the mean field was strongly gauge dependent, but that from the fluctuating field was not.

T. Rogers: To calculate magnetic helicity you assumed that the solar wind was isotropic, which observations show it is not. How would this affect the results you present?

A. Brandenburg: Since we have to adopt the Taylor hypothesis, only the two magnetic field components perpendicular to the radial direction enter the calculation. The field in the plane perpendicular to the radial direction is still fairly isotropic, so I guess our results are still meaningful. To clarify the significance of our results further, it might be useful to compute magnetic helicity from simulations of anisotropic MHD turbulence with one preferred direction.

D. Hughes: In your simulations that show the generation of large-scale fields on a long time, what is the timescale for the generation of the fields? If it is ohmic then it is not surprising.

A. Brandenburg: The initial exponential growth occurs always on a dynamical time scale, but full saturation is only obtained on a resistive time scale. We know that this problem can only be alleviated by magnetic helicity fluxes, which are quite weak in our Cartesian simulations. Nevertheless, our simulations prove the point that the $\alpha$ effect works in rotating convection, which was until now quite unclear.

C. Forest: How do you model the boundary conditions? Open or Closed?

A. Brandenburg: At the bottom we adopt a perfect conductor boundary condition and at the top we assume that the horizontal field vanishes. This pseudo-vacuum condition is numerically more robust than a proper vacuum condition. However, it would be more realistic to couple the convection simulation to a force-free model, as has been done for forced turbulence simulations in the paper by Warnecke \& Brandenburg (2010), which is also presented here as a poster. 